\title{
Article
}

\section{An Extension of the Bland-Altman Plot for Analyzing the Agreement of More than Two Raters}

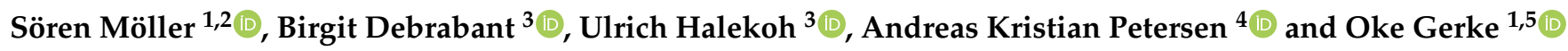 \\ 1 Department of Clinical Research, University of Southern Denmark, 5000 Odense C, Denmark; \\ moeller@health.sdu.dk (S.M.); Oke.Gerke@rsyd.dk (O.G.) \\ 2 Open Patient data Explorative Network, Odense University Hospital, 5000 Odense C, Denmark \\ 3 Department of Public Health, Epidemiology, Biostatistics and Biodemography, University of Southern \\ Denmark, 5000 Odense C, Denmark; bdebrabant@health.sdu.dk (B.D.); uhalekoh@health.sdu.dk (U.H.) \\ 4 Department of Research and Learning, Hospital of Southern Jutland, 6200 Aabenraa, Denmark; \\ Andreas.Kristian.Pedersen@rsyd.dk \\ 5 Department of Nuclear Medicine, Odense University Hospital, 5000 Odense C, Denmark
}

check for updates

Citation: Möller, S.; Debrabant, B.; Halekoh, U.; Petersen, A.K.; Gerke, O. An Extension of the Bland-Altman Plot for Analyzing the Agreement of More than Two Raters. Diagnostics 2021, 11, 54. https://doi.org/ 10.3390/diagnostics11010054

Received: 6 November 2020 Accepted: 28 December 2020 Published: 1 January 2021

Publisher's Note: MDPI stays neutral with regard to jurisdictional clai$\mathrm{ms}$ in published maps and institutional affiliations.

Copyright: (C) 2021 by the authors. Licensee MDPI, Basel, Switzerland. This article is an open access article distributed under the terms and conditions of the Creative Commons Attribution (CC BY) license (https:// creativecommons.org/licenses/by/ $4.0 /)$.

\begin{abstract}
The Bland-Altman plot is the most common method to analyze and visualize agreement between raters or methods of quantitative outcomes in health research. While very useful for studies with two raters, a limitation of the classical Bland-Altman plot is that it is specifically used for studies with two raters. We propose an extension of the Bland-Altman plot suitable for more than two raters and derive the approximate limits of agreement with $95 \%$ confidence intervals. We validated the suggested limit of agreement by a simulation study. Moreover, we offer suggestions on how to present bias, heterogeneity among raters, as well as the uncertainty of the limits of agreement. The resulting plot could be utilized to investigate and present agreement in studies with more than two raters.
\end{abstract}

Keywords: Bland-Altman plot; agreement; visualization; simulation study; method comparison; inter-rater; intra-rater

\section{Introduction}

In health research, it is often desired to determine to what degree different raters or methods (either persons or devices) agree on the measurement of a continuous outcome on the same subject (patient, diagnostic image, biological sample, etc.). The main aim is to ensure that the variability between raters is small enough to use observations from a single rater in future studies or clinical practice and that it does not make any difference which rater conducted the single measurement.

Typically this situation will be dealt with by applying a mixed effect regression model or related methods [1-3]. In addition to these quantitative results, a clinical researcher often wishes to present the agreement graphically, to ease the interpretation and communication of the results. The most common method used for this aim is the Bland-Altman (BA) plot [4], which plots differences between two raters against respective means together with $95 \%$ limits of agreement (LOAs). The interpretation and reporting of these LOAs regularly results in confusion; see [5-7] for recent overviews.

One of the drawbacks of the classical Bland-Altman plot is that it only applies to a situation with two raters or methods, while for both practical reasons (distributing workload to multiple raters) and statistical reasons (increased power and strengthened generalizability), it is desirable to use more than two raters for carrying out agreement studies. The standard suggestion is usually to present multiple Bland-Altman plots for each pairwise comparison of raters $[3,8]$, but this becomes awkward for more than four raters, as $m$ raters result in $m \cdot(m+1) / 2$ plots, which is difficult to present and cumbersome to interpret. Moreover, the pairwise nature of the limit band in this situation restricts the interpretability of the bands. 
Some suggestions include presenting all observations in the same plot, with one marker for each observation instead of each subject, either connecting observations for the same subject by lines [3] or by different marker symbols [9]. However, both of these approaches become confusing when including many observations. Other suggested approaches decrease the number of plots to $m-1$ under the assumption of one method being a gold standard [10] or reduce the presentation to one plot, but being much different in appearance from a classical BA plot and removing the concept of an LOA, which can be compared with prespecified levels of clinically meaningful differences [11].

The aim of this paper is to present a novel extension of the classical BA plot together with a generalized LOA, which only requires one plot and one marker per observed subject. While the main aim of this proposal is the comparison of multiple raters, the plot can be applied to comparisons of multiple methods as well. Especially for this case, it is important to be aware of the assumption of homoscedasticity, which our proposal inherits from the classical Bland-Altman plot; deviations from this assumption damage the interpretation of the Bland-Altman limits of agreement $[12,13]$.

\section{Materials and Methods}

\subsection{Derivation of an LOA for $m>2$ Raters}

We are interested in investigating a situation with $m$ raters who observe each of $n$ subjects once, resulting in $m \cdot n$ observations $x_{i, j}$.

In this setting, we assume a data generating process similar to the assumptions made by Bland and Altman [4] for the classical BA plot, but extended to more than 2 raters:

$$
X_{i, j}=\mu_{i}+\gamma_{j}+\epsilon_{i, j} \quad i=1, \ldots, n, j=1, \ldots, m .
$$

Each subject's true value $\mu_{i}$ and each rater's bias $\gamma_{j}$ are deterministic, and the measurement error term $\epsilon_{i, j} \sim N\left(0, \sigma^{2}\right)$ determines the variation in the observations, assuming that all $\epsilon_{i, j}$ are pairwise independent with constant variance.

In the classical Bland-Altman plot (for $m=2$; see the lower row of Figure 1), the points:

$$
\left(\bar{x}_{i}, d_{i}\right) \stackrel{\text { def }}{=}\left(\frac{x_{i, 1}+x_{i, 2}}{2}, x_{i, 1}-x_{i, 2}\right), \quad i=1, \ldots, n
$$

are calculated and plotted together with a bias line at:

$$
\bar{d} \stackrel{\text { def }}{=} \frac{1}{n} \sum_{i=1}^{n} d_{i}=\frac{1}{n} \sum_{i=1}^{n} x_{i, 1}-\frac{1}{n} \sum_{i=1}^{n} x_{i, 2}
$$

and LOA at:

$$
(U, L) \stackrel{\text { def }}{=} \bar{d} \pm t_{0.975, n-1} \cdot \sqrt{1+\frac{1}{n}} \cdot s_{d}
$$

Here:

$$
s_{d} \stackrel{\text { def }}{=} \sqrt{\frac{1}{n-1} \sum_{i=1}^{n}\left(d_{i}-\bar{d}\right)^{2}}
$$

is the empirical standard deviation of the observed differences. Note that the LOA corresponds to a $95 \%$ prediction interval for a new Gaussian distributed difference.

Instead of $t_{0.975, n-1} \cdot \sqrt{1+\frac{1}{n}}$, approximations are applied in practice, mostly either 1.96 , the large $n$ limit, or the approximation 2 . Usually, the term $\sqrt{1+\frac{1}{n}}$ is dropped (see $[3,8]$ for discussions on this issue). In this paper, we apply the exact factor $t_{0.975, n-1} \cdot \sqrt{1+\frac{1}{n}}$ in the derivation of the classical BA LOA [10] due to our later investigations into small sample sizes such as $n=10$. 
For the case of $m>2$ raters, we suggest plotting:

$$
\left(\bar{x}_{i}, s_{i}\right) \stackrel{\text { def }}{=}\left(\frac{1}{m} \sum_{j=1}^{m} x_{i, j}, \sqrt{\frac{1}{m-1} \sum_{j=1}^{m}\left(x_{i, j}-\bar{x}_{i}\right)^{2}}\right), \quad i=1, \ldots, n,
$$

resulting in an $\mathrm{x}$-axis corresponding to the classical Bland-Altman plot, but with the $\mathrm{y}$ axis presenting the intra-subject standard deviation instead of the observed differences, resulting in only one marker for each observed subject even with more than 2 raters. This is in accordance with a suggestion made earlier by Bland and Altman [4], but not yet widely applied. Note that this change mathematically corresponds to switching from plotting an $L_{1}$ distance on the y-axis to plotting an $L_{2}$ distance instead.

If $m=2$, this results in a plot corresponding to the classical Bland-Altman plot in which the points of the lower half of the BA plot are mirrored at the bias line and the $y$ axis is scaled by a factor of $\sqrt{2}$. Figure 1 contrasts our suggestion with the classical Bland-Altman plot for $m=2$, while Figure 2 gives examples of our plot for $m>2$ and different choices of $n$.
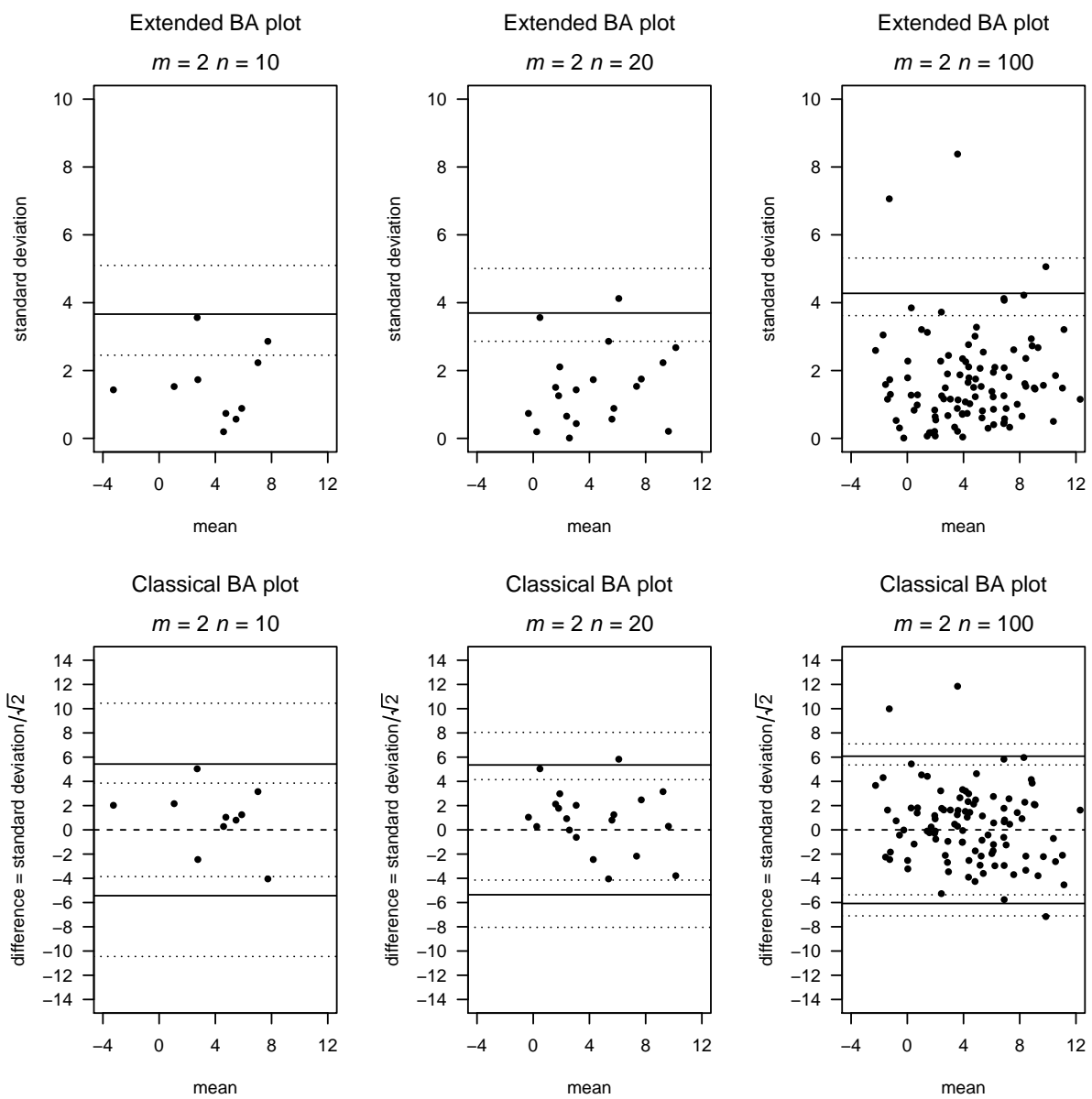

Figure 1. Comparison between the extended and classical BA plot for 2 raters based on simulated data with a true standard deviation $\sigma=2$.

While it is intuitive where the sample should be placed, the placement of a 95\% LOA requires some additional considerations. Here, we use the property that the empirical variance of $m$ independent, normally distributed random variables with equal mean (hence, excluding systematic bias between raters) and constant variance is $\chi^{2}$-distributed with $m-1$ degrees of freedom, and hence, the empirical standard deviation will be $\chi$-distributed with $m-1$ degrees of freedom, ignoring appropriate scaling factors. 

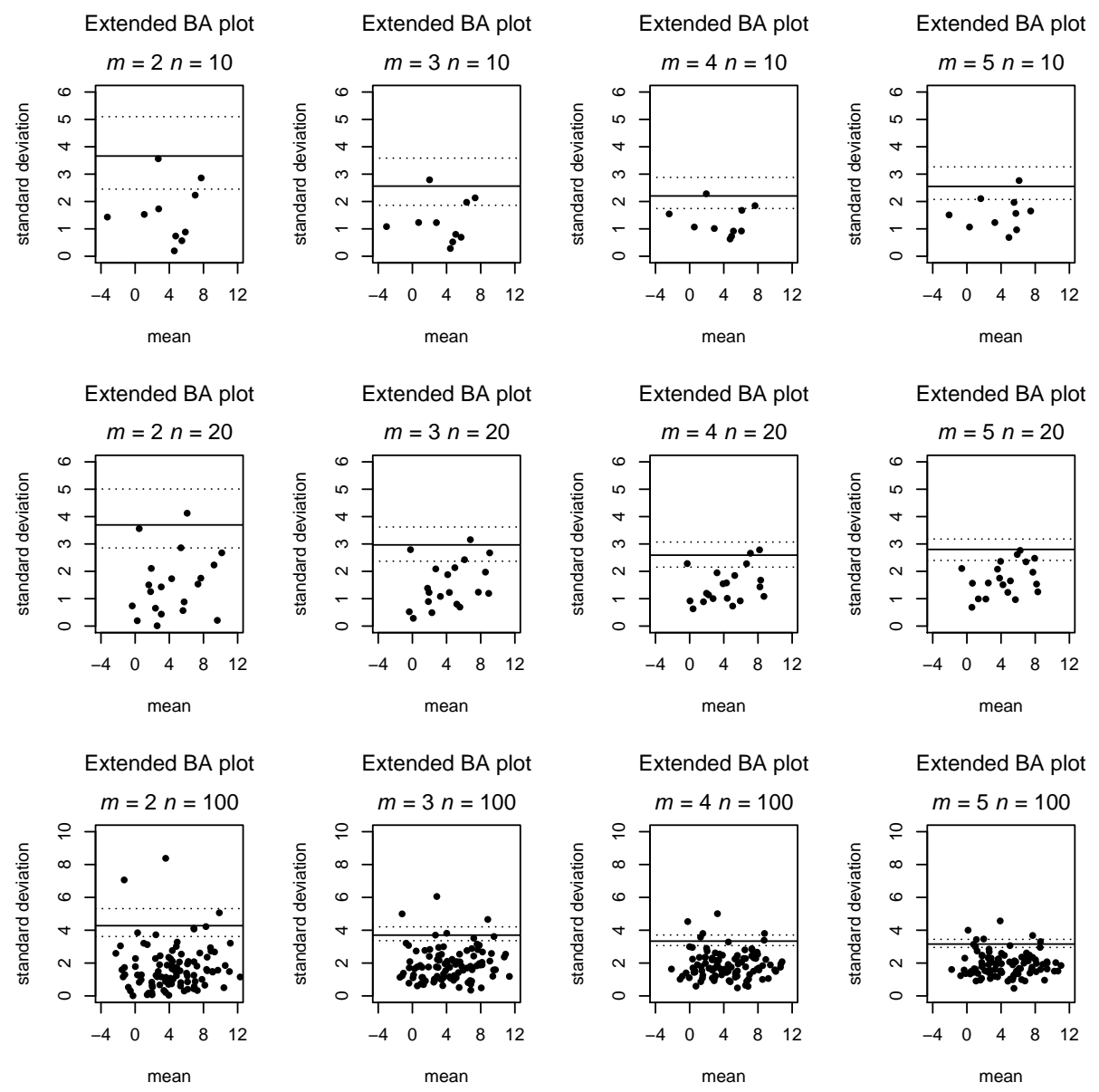

Figure 2. Extended BA plot for varying number of raters and observed subjects based on simulated data.

Applying this to our model, we observe asymptotically:

$$
\frac{s_{i}}{\sigma} \sqrt{m-1} \sim \chi(m-1) \quad i=1 \ldots n, j=1 \ldots m .
$$

Therefore, we propose to place our LOA at:

$$
L \stackrel{\text { def }}{=} \chi_{0.95, m-1} \frac{1}{\sqrt{m-1}} S
$$

where $\chi_{0.95, m-1}$ denotes the quantile function of a $\chi$-distribution with $m-1$ degrees of freedom and $s=n^{-1} \sum_{i}^{n} s_{i}$ is the average intra-subject standard deviation. Investigating agreement between methods or raters, this LOA then can be compared to a clinically relevant difference by rescaling the y-axis with a factor of $\sqrt{2}$, which enables direct comparison of deviations from the different ratings as used in our method with differences of 2 ratings as used in classical BA plots. We decided not to propose this rescaling in general for the extended plots, as the rescaled y-axis loses its clear statistical interpretation as a within-subject standard deviation.

Note that this formula only takes into account the number of raters, but neither the number of subjects observed (that is the uncertainty in $s$, which in the classical BlandAltman plot is taken into account by using the $t$-quantile), nor that the coverage achieved by the LOA should correspond to a $95 \%$ prediction interval for an additional observation instead of covering $95 \%$ of the original observations [14] (the factor $\sqrt{1+1 / n}$ in the classical Bland-Altman plot); hence, our formula is only expected to be precise for large $n$. To communicate the uncertainty of the LOA, we suggest plotting $95 \%$ confidence 
intervals around the line. Here, we did this by applying bootstrapping in each sample with 1000 repetitions and reporting a 95\% bias-corrected and accelerated confidence interval. For comparison, we plot the exact confidence intervals suggested by Carkeet [15] in the classical BA plots presented in this paper.

\subsection{Suggestion for Indicating Bias}

One limitation of our suggested plot is losing the information on a possible bias of a specific rater, as well as possible heterogeneity between the uncertainty of raters. To include information on this phenomenon in the plot, we suggest adding tick marks on the y-axis corresponding to:

$$
\left|B_{j}\right| \stackrel{\text { def }}{=}\left|\frac{1}{N} \sum_{i=1}^{n} x_{i, j}-\bar{x}_{i}\right| \quad i=1, \ldots n, j=1, \ldots m,
$$

the absolute mean difference between rater $j$ 's observation and each subjects average observation. Moreover, we suggest marking each point in the plot by a color (or symbol) to distinguish which rater was responsible for the largest deviation from this subject's mean; hence, a color/symbol very common in the plot would indicate a rater with larger uncertainty than the remaining raters, violating the assumption of $\sigma$ not depending on $j$. Figure 3 shows these additions in a scenario without bias, a scenario in which Rater 2 systematically measures, on average, one standard deviation above the true value, and a scenario in which Rater 2 has much larger uncertainty than the remaining raters. Moreover, Figure 4 presents a scenario in which variability increases with the true mean, corresponding to the funnel shape appearing in a classical BA plot. The full algorithm for preparing an extended Bland-Altman plot is presented as Algorithm 1.

Algorithm 1 EXTENDED BLAND-ALTMAN PLOT FOR MULTIPLE RATERS.

Observe number of raters $m$ and number of subjects $n$ and individual ratings

$$
x_{i, j} \quad i=1 \ldots n, j=1 \ldots m
$$

Determine each subject's mean and standard deviation:

$$
\left(\bar{x}_{i}, s_{i}\right)=\left(\frac{1}{m} \sum_{j=1}^{m} x_{i, j} \sqrt{\frac{1}{m-1} \sum_{j=1}^{m}\left(x_{i, j}-\bar{x}_{i}\right)^{2}}\right), \quad i=1, \ldots, n,
$$

Determine the position of the LOA:

$$
L=\chi_{0.95, m-1} \frac{1}{\sqrt{m-1}} s \quad \text { with } s=n^{-1} \sum_{i}^{n} s_{i} .
$$

Estimate the $95 \%$ confidence interval for the LOA by bootstrapping Determine bias indicators for each rater:

$$
\left|B_{j}\right|=\left|\frac{1}{N} \sum_{i=1}^{n} x_{i, j}-\bar{x}_{i}\right| \quad i=1 \ldots n, j=1 \ldots m,
$$

Plot $\left(\bar{x}_{i}, s_{i}\right), L$ with the confidence interval, and $\left|B_{j}\right|$ for all raters in a figure 
Extended BA plot

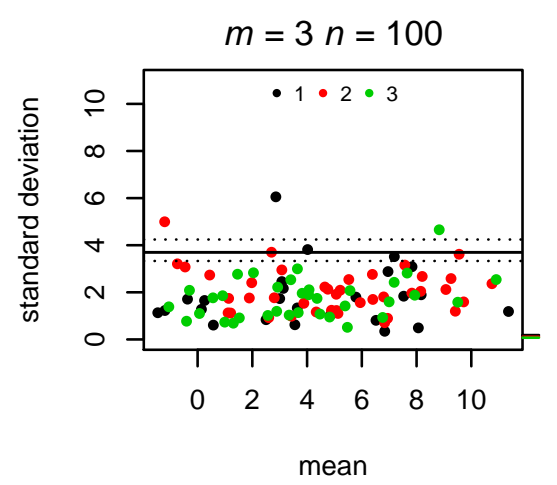

Extended BA plot

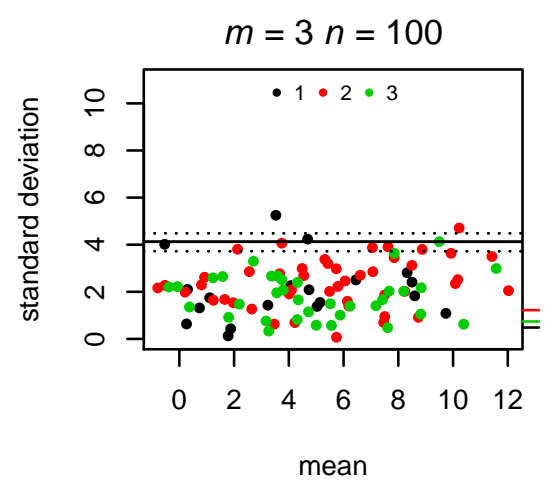

Extended BA plot

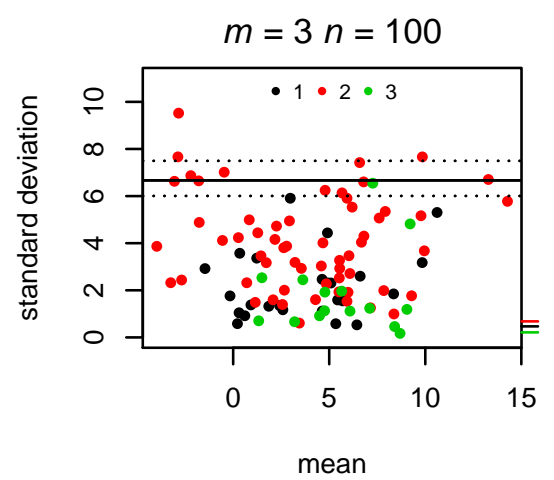

Extended BA plot

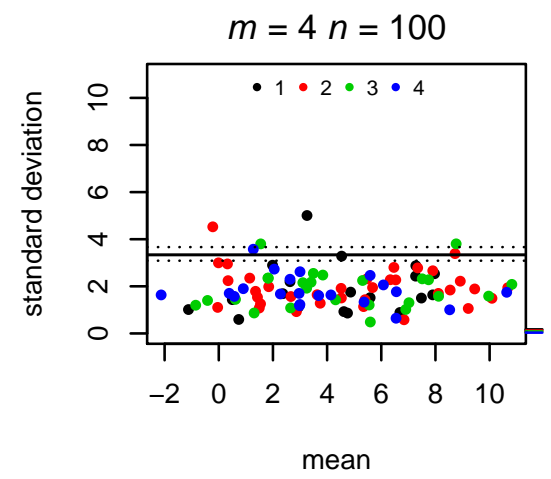

Extended BA plot

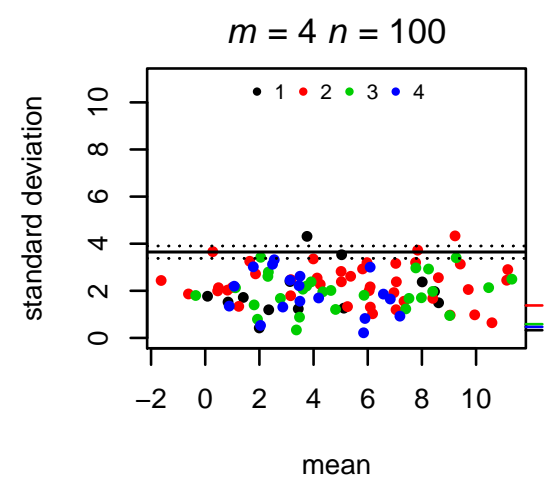

Extended BA plot

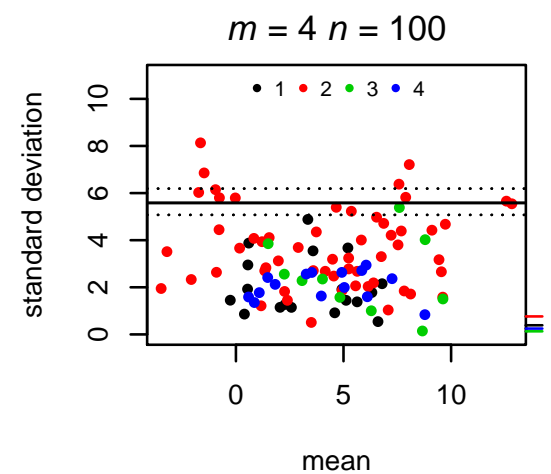

Extended BA plot

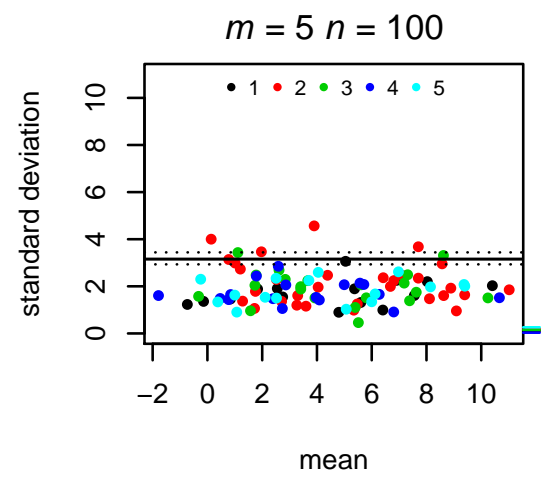

Extended BA plot

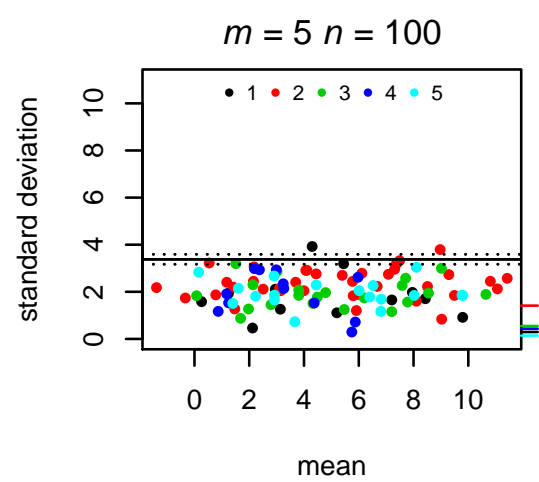

Extended BA plot

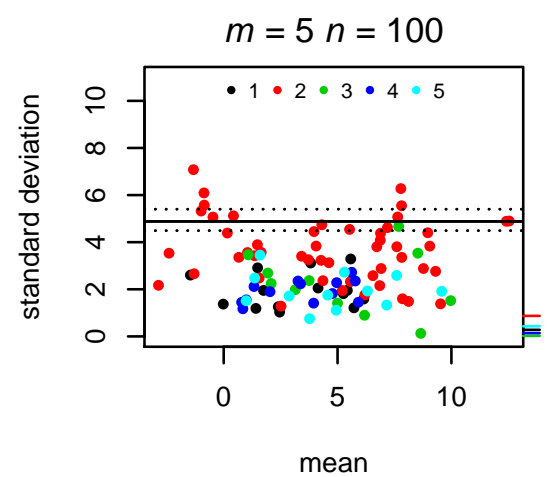

Figure 3. Marking the rater responsible for the largest deviation from the intra-subject mean by colored dots and the absolute rater bias by tick marks on the right y axis. The first row demonstrates absent bias and heterogeneity in error, the second row only bias, and the third row only heterogeneity $(\sigma=\sqrt{40}$ for Rater 2 in the third row; all others $\sigma=2)$. 

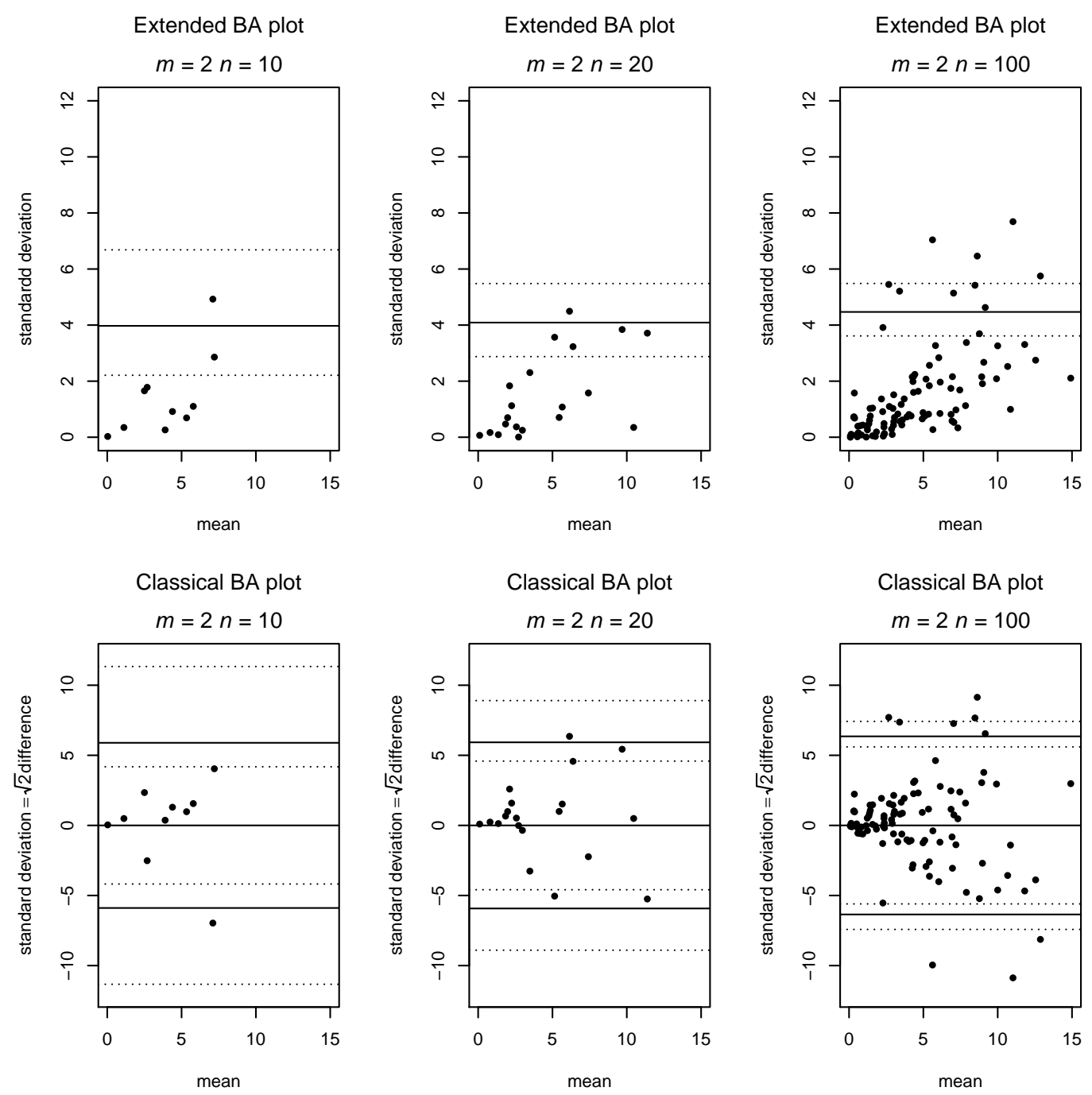

Figure 4. Comparison between the classic and generalized BA plot in a situation where the measurement error increases with the true value.

\subsection{Simulation Study on Coverage}

To determine how reasonably our LOA agrees with the desired nominal 95\% coverage level for one new observation, we carried out a simulation. We employed 10,000 simulated samples, as this, in the worst case scenario, should result in Monte Carlo SE below 0.5\% according to Morris et al. [16]. Data were generated by the process:

$$
X_{i, j}=\mu_{i}+\epsilon_{i, j} \quad i=1, \ldots, n+1, j=1, \ldots, m,
$$

that is without systematic bias, for each combination of $m=2,3,4,5$ raters and $n=10,20,100$ subjects. For each of these 12 combinations, we determined the coverage of the LOA based on simulated data. Furthermore, we calculated the empirical $95 \%$ quantile of points, both for the original $n$ observations used to produce the plot and for one new observation $(i=n+1)$ from the same process for each sample.

$R$ Version 3.6.1 [17] with the packages nnet [18] and boot [19,20] was used to carry out the simulations and produce the figures. $\mathrm{R}$ scripts, including the seeds applied in the simulations, are available as Supplementary Files. 


\section{Results}

\subsection{Simulation Results}

Figure 1 compares the classic and extended Bland-Altman plots in the case of two raters with simulated data. Figure 2 shows examples of generalized Bland-Altman plots for varying numbers of raters and observed subjects. Table 1 shows the empirical $95 \%$ LOA compared with the line suggested by our Formula (1), as well as the coverage obtained by applying the LOA of our formula. It can be seen that the coverage generally is between 0.92 and 0.96 , although most typically slightly below 0.95 . We generally see a coverage slightly above $95 \%$ and a narrower LOA than expected from the formula for the original observations. On the other hand, for the new observations, the coverage is slightly below $95 \%$, and the empirical quantile is slightly wider than the LOA, although the obtained coverage of 93 to $95 \%$ is deemed acceptable. This decreased coverage is expected, as our formula for the LOA does not take into account the additional uncertainty of the new observations.

Table 1. Coverage and empirical 95\% quantiles from the simulations of different choices of $m$ and $n$. Results are reported both for the original observations used to estimate the LOA, as well as for the new observations obtained from the same data generating process.

\begin{tabular}{lccccccc}
\hline & & \multicolumn{3}{c}{ Original Observations } & \multicolumn{3}{c}{ New Observations } \\
\hline & & $\boldsymbol{n = 1 0}$ & $\boldsymbol{n = 2 0}$ & $\boldsymbol{n = 1 0 0}$ & $\boldsymbol{n = 1 0}$ & $\boldsymbol{n = 2 0}$ & $\boldsymbol{n = 1 0 0}$ \\
\hline Raters & Formula (1) & $\mathbf{9 5 \%}$ Quantile & $\mathbf{9 5 \%}$ Quantile & $\mathbf{9 5 \%}$ Quantile & $\mathbf{9 5 \%}$ Quantile & $\mathbf{9 5 \%}$ Quantile & $\mathbf{9 5 \%}$ Quantile \\
\hline$m=2$ & 1.959964 & 1.907143 & 1.939001 & 1.954703 & 2.188023 & 2.069437 & 1.963274 \\
$m=3$ & 1.730818 & 1.681992 & 1.709057 & 1.726664 & 1.880320 & 1.780882 & 1.741569 \\
$m=4$ & 1.613973 & 1.572103 & 1.609547 & 1.612125 & 1.706937 & 1.654243 & 1.624840 \\
$m=5$ & 1.540108 & 1.525253 & 1.536958 & 1.534751 & 1.594541 & 1.57577 & 1.552975 \\
\hline & & Coverage & Coverage & Coverage & Coverage & Coverage & Coverage \\
\hline$m=2$ & 0.9575 & 0.9528 & 0.9506 & 0.9247 & 0.9400 & 0.9497 \\
$m=3$ & 0.9594 & 0.9542 & 0.9507 & 0.9253 & 0.9404 & 0.9488 \\
$m=4$ & 0.9595 & 0.9539 & 0.9510 & 0.9297 & 0.9417 & 0.9479 & 0.9462 \\
$m=5$ & 0.9597 & 0.9541 & 0.9508 & 0.9361 & & 0.9408 &
\end{tabular}

Figure 3 shows the indications of bias in a single observer, as well as increased uncertainty in a single rater compared to a situation without these phenomena. In the second row (with bias), it can clearly be seen that Rater 2's tick mark is elevated compared to the other raters, while in the third row (with increased measurement error), the plot is dominated by red points, corresponding to Rater 2 most often being further away from the intra-subject mean. Hence, both deviations from model assumptions can be detected and distinguished by the extended BA plot with bias tick marks and color coded observations.

Figure 4 presents a situation in which the assumption of homogenous variance is violated, as the measurement error increases with increasing true mean. Again, we compare classic and extended Bland-Altman plots on the same data, and the inhomogeneity of variance is clearly visible as a funnel shape in both plots.

\subsection{Application to Real World Data}

To investigate the usefulness of the extended BA plot, we applied it to two datasets with multiple raters from the literature. For the first application (Figure 5 left), we used data from Wiinholt et al. [21] corresponding to measurements of tissue volume in 30 control mice by four raters. Our plot corresponds to the results presented in the left side of Figure 3 of [21] as six separate BA plots. Our plot indicates an LOA around 0.85 with a $95 \%$ up to almost one and no clear bias, but possibly a tendency of Method 4 to be more variable compared to the other methods.

For the second application, we applied the extended BA plot to the glucose measurement data from a method comparison study [22] with four methods as provided in the MethComp [23] package for R (Figure 5 right). Our plot indicates an LOA around 1.2 
with a narrow confidence interval and no clear indication of either bias or heterogeneity of variance.

Wiinholt et al.

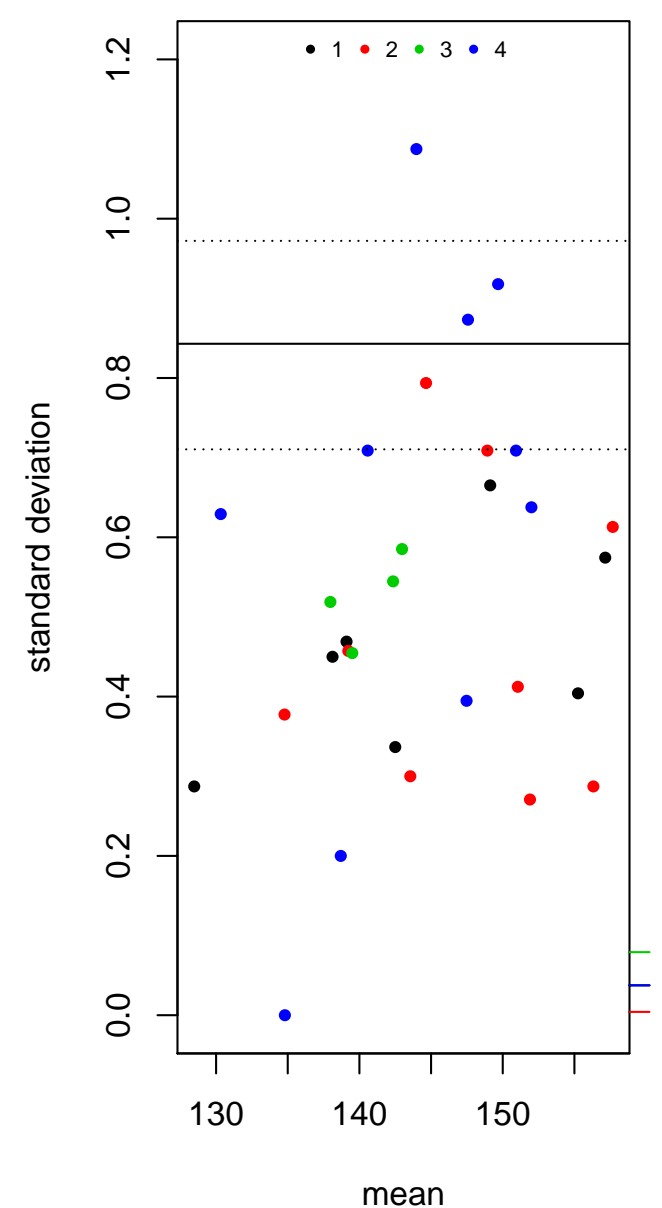

Carstensen et al.

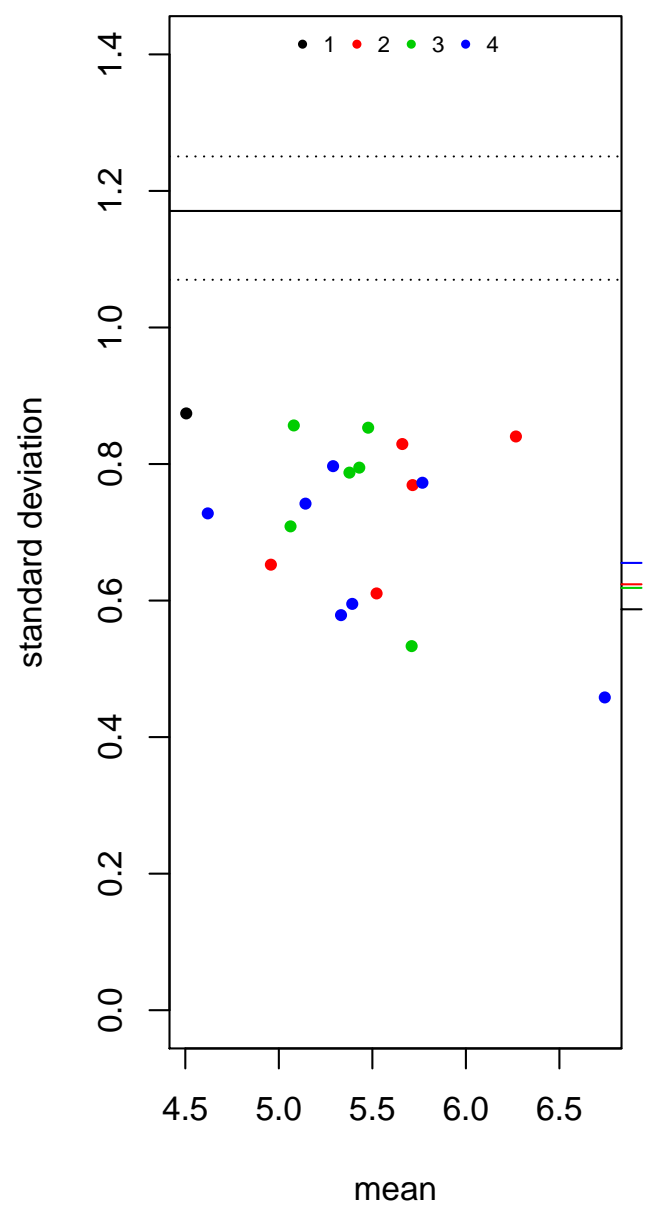

Figure 5. Extended BA plot on real-world data. Wiinholt et al. [21] (left) and Carstensen et al. [22] (right).

\section{Discussion}

\subsection{Statement of Principal Findings}

We showed that it is possible to present the agreement of more than two methods or raters in one combined plot and to obtain similar information as can be obtained from a classical BA plot including LOA and the corresponding confidence intervals; moreover, we indicated the possibility of detecting inhomogeneity in variance, as well as bias among raters. We showed by simulation that our suggested formula for the LOA of the extended BA obtains approximately $95 \%$ coverage.

\subsection{Strengths and Weaknesses of the Study}

The main strength of this study is that we combined a closed formula for the LOA based on mathematical arguments with transparent simulations documenting that the method behaves well even with small sample sizes. Moreover, we showed that our suggested plot can combine information presented in multiple plots in applications from the literature. A weakness of this study is that the LOA is only an approximation, as it does not fully capture the uncertainty of new observations, as well as only being equipped with approximate bootstrapped confidence intervals. 


\subsection{Strengths and Weaknesses in Relation to Other Studies, Particularly any Differences in the Results}

Compared to the literature, the main strength of our approach is that only one plot is required for the comparison of multiple methods or raters and that only one dot is included in the plot for each observation unit, resulting in plots that are easier to comprehend. Moreover, these facts imply that the points on our plot are independent observations facilitating the estimation of accurate LOA and confidence intervals. Although it is possible to indicate bias and variance heterogeneity in our plot, it might be less clear than in some of the methods presented in the literature. Additionally, as our plot is only one-sided due to the non-negativity of standard deviations, this might impede its interpretation for readers used to classical BA plots.

An additional weakness of the proposed method is that it shares the assumption of homoscedasticity with the classical BA plot. It is well known from the literature that this assumption can be problematic, especially in the case of method comparison studies [12,13]. There have been detailed proposals of replacements of the classical BA plot, which handle heteroscedasticity in a more suitable manner both theoretically $[24,25]$ and with practical applications and software implementations [26-28]. We consider this problem to be mainly prominent in the case of method comparison studies and to a lesser degree when comparing raters. As the suggested approach by Taffé (similarly to the classical BA plot) only handles comparison of two methods, we consider our proposal to be a relevant extension of the classical BA plot in the case of multiple raters and homoscedasticity.

\subsection{Meaning of the Study: Possible Mechanisms and Implications for Clinicians or Policymakers}

The possibility to present agreement information for more than two raters hopefully increases the possibility to report this information in agreement studies and to better inform the readers. Indirectly, this also might increase the inclination to include multiple raters in agreement studies, improving the generalizability of such studies. This implies that researchers can include the same observations in a graphical presentation as in more advanced analyses in studies with multiple raters, e.g., mixed models, improving the transparency of the studies. The fact that our LOA is presented with $95 \%$ confidence bands hopefully increases the likelihood that users of this plot will include confidence bands as well, which still is not the case in many papers published with classical BA plots [5,29]. One of the challenges for the extended BA plot, as well as for most other suggested extensions or replacements of the classical BA plot, is the inertia in health research of replacing commonly used methods (think, for instance, of scatter plots of two methods' raw measurements with a 45 degree line and respective correlation coefficients back in the 1970s). We hope that our suggested plot is similar enough to the classical BA plot to aid its adoption in practice.

\subsection{Unanswered Questions and Future Research}

There are still some outstanding questions with regard to our suggested extension of the BA plot. Firstly, it would be desirable to obtain a more precise, non-asymptotic formula for the LOA taking into account the full amount of uncertainty in the observations. Secondly, a closed formula for the confidence interval would be preferable to our bootstrap approach. Finally, implementations of our plot in standard software packages will improve its applicability.

Supplementary Materials: The following are available online at https:/ /www.mdpi.com/2075-441 8/11/1/54/s1: Simulation.R: Source code for the simulations, Figures.R: Source code for Figures 1-4, Examples.R: Source code for real-world examples, Figure 5.

Author Contributions: Conceptualization, all authors; software, S.M.; writing, original draft preparation, S.M.; writing, review and editing, all authors; visualization, S.M. All authors read and agreed to the published version of the manuscript.

Funding: This research received no external funding. 
Data Availability Statement: The simulated data presented in this study are reproducible by the $\mathrm{R}$ code in the supplementary material (Simulation.R). The real word example data is available in publicly accessible repositories, as referenced in the supplementary material (Examples.R).

Conflicts of Interest: The authors declare no conflict of interest.

\author{
Abbreviations \\ BA plot Bland-Altman plot \\ LOA limit of agreement
}

The following abbreviations are used in this manuscript:

\title{
References
}

1. Gerke, O.; Möller, S.; Debrabant, B.; Halekoh, U. Experience from applying the Guidelines for Reporting Reliability and Agreement Studies (GRRAS) indicated 5 questions to be addressed in the planning phase from a statistical point of view. Diagnostics 2018, 8, 69. [CrossRef]

2. Carstensen, B. Comparing and predicting between several methods of measurement. Biostatistics 2004, 5, 399-413. [CrossRef] [PubMed]

3. Carstensen, B. Comparing Clinical Measurement Methods; Wiley: Hoboken, NJ, USA, 2010.

4. Bland, J.M.; Altman, D.G. Statistical methods for assessing agreement between two methods of clinical measurement. Lancet 1986, 327, 307-310. [CrossRef]

5. Abu-Arafeh, A.; Jordan, H.; Drummond, G. Reporting of method comparison studies: A review of advice, an assessment of current practice, and specific suggestions for future reports. Br. J. Anaesth 2016, 117, 569-575. [CrossRef] [PubMed]

6. Flegal, K.M.; Graubard, B.; Ioannidis, J.P.A. Use and reporting of Bland-Altman analyses in studies of self-reported versus measured weight and height. Int. J. Obes. 2019, 44, 1311-1318. [CrossRef]

7. Gerke, O. Reporting Standards for a Bland-Altman Agreement Analysis: A Review of Methodological Reviews. Diagnostics 2020, 10, 334. [CrossRef]

8. Bland, J.M.; Altman, D.G. Measuring agreement in method comparison studies. Stat. Methods Med. Res. 1999, 8, 135-160. [CrossRef]

9. Jones, M.; Dobson, A.; O’Brian, S. A graphical method for assessing agreement with the mean between multiple observers using continuous measures. Int. J. Epidemiol. 2011, 40, 1308-1313. [CrossRef]

10. Proschan, M.A.; Leifer, E.S. Comparison of two or more measurement techniques to a standard. Contemp. Clin. Trials 2006, 27, 472-482. [CrossRef]

11. Scott, L.E.; Galpin, J.S.; Glencross, D.K. Multiple method comparison: Statistical model using percentage similarity. Cytom. B Clin. Cytom. 2003, 54, 46-53. [CrossRef]

12. Taffé, P. Effective plots to assess bias and precision in method comparison studies. Stat. Methods Med. Res. 2018, 27, 1650-1660. [CrossRef] [PubMed]

13. Carstensen, B. Comparing methods of measurement: Extending the LoA by regression. Stat. Med. 2010, 29, 401-410. [CrossRef] [PubMed]

14. Vock, M. Intervals for the assessment of measurement agreement: Similarities, differences, and consequences of incorrect interpretations. Biom. J. 2016, 58, 489-501. [CrossRef] [PubMed]

15. Carkeet, A. Exact parametric confidence intervals for Bland-Altman limits of agreement. Optom. Vis. Sci. 2015, 92, 71-80. [CrossRef] [PubMed]

16. Morris, T.P.; White, I.R.; Crowther, M.J. Using simulation studies to evaluate statistical methods. Stat. Med. 2019, 38, 2074-2102. [CrossRef] [PubMed]

17. R Core Team. R: A Language and Environment for Statistical Computing; R Foundation for Statistical Computing: Vienna, Austria, 2020.

18. Venables, W.N.; Ripley, B.D. Modern Applied Statistics with S, 4th ed.; Springer: New York, NY, USA, 2002; ISBN 0-387-95457-0.

19. Canty, A.; Ripley, B.D. boot: Bootstrap R (S-Plus) Functions, R package version 1.3-24, 2019. Available online: https:/ / cran.rproject.org/web/packages/boot/ (accessed on 31 December 2020).

20. Davison, A.C.; Hinkley, D.V. Bootstrap Methods and Their Applications; Cambridge University Press: Cambridge, UK, 1997; ISBN 0-521-57391-2.

21. Wiinholt, A.; Gerke, O.; Dalaei, F.; Bučan, A.; Madsen, C.B.; Sørensen, J.A. Quantification of tissue volume in the hindlimb of mice using microcomputed tomography images and analysing software. Sci. Rep. 2020, 10, 8297. [CrossRef]

22. Carstensen, B.; Lindström, J.; Sundvall, J.; Borch-Johnsen, K.; Tuomilehto, J.; Aunola, S.; Cepaitis, Z.; Eriksson, J.; Hakumäki, M.; Hämäläinen, H.; et al. Measurement of blood glucose: Comparison between different types of specimens. Ann. Clin. Biochem. 2008, 45, 140-148. [CrossRef]

23. Carstensen, B.; Gurrin, L.; Ekstrøm, C.T.; Figurski, M. MethComp: Analysis of Agreement in Method Comparison Studies, R package version 1.30.0, 2020. Available online: https://rdrr.io/cran/MethComp/ (accessed on 31 December 2020). 
24. Nawarathna, L.S.; Choudhary, P.K. Measuring agreement in method comparison studies with heteroscedastic measurements. Stat. Med. 2013, 32, 5156-5171. [CrossRef]

25. Nawarathna, L.S.; Choudhary, P.K. A heteroscedastic measurement error model for method comparison data with replicate measurements. Stat. Med. 2015, 34, 1242-1258. [CrossRef]

26. Taffé, P.; Peng, M.; Stagg, V.; Williamson, T. Method Compare: An R package to assess bias and precision in method comparison studies. Stat. Methods Med. Res. 2019, 28, 2557-2565. [CrossRef]

27. Taffé, P.; Peng, M.; Stagg, V.; Williamson, T. biasplot: A package to effective plots to assess bias and precision in method comparison studies. Stat. J. 2017, 17, 208-221. [CrossRef]

28. Taffé, P.; Halfon, P.; Halfon, M. A new statistical methodology overcame the defects of the Bland-Altman method. J. Clin. Epidemiol. 2020, 124, 1-7. [CrossRef] [PubMed]

29. Chhapola, V.; Kanwal, S.K.; Brar, R. Reporting standards for Bland-Altman agreement analysis in laboratory research: A crosssectional survey of current practice. Ann. Clin. Biochem. 2015, 52, 382-386. [CrossRef] [PubMed] 